\title{
ELEMENTOS TEÓRICOS DEL CUIDADO DE ENFERMERÍA EN UCI
}

Miladys Gallego*, Milena Leal*, Ximena Ramos*

\section{Resumen}

Investigación cualitativa de tipo descriptivo que pretende identificar los elementos teóricos del cuidado de enfermería en las UCI de dos instituciones hospitalarias de Bogotá. Se realizó entre julio de 2006 y noviembre de 2007 y la muestra la conformaron las ENF que laboraban allí. La información se obtuvo de una entrevista semiestructurada grabada en audio y la cantidad de participantes se determinó por principio de saturación. Los resultados obtenidos son las respuestas de las ENF entrevistadas sobre el cuidado de enfermería, quienes lo definen como la atención directa que se presta a un ser holístico, cuyo fin es la satisfacción de necesidades siendo muy prioritarias las de origen fisiológico. Seis ENF refieren haber conocido los modelos y teorías durante su formación universitaria, cuatro mencionaron alguna autora o conceptos propios de una teoría y solo una demostró dominio básico de una de las teorías. Tres ENF refirieron desconocimiento total sobre el tema. Esta investigación hace pensar que las ENF no hacen uso metódico de los modelos y teorías, su práctica no encuentra fundamento importante en estos y muy pocas se interesan en su estudio y aplicación formal. Sin embargo, se identifican en las respuestas de las entrevistadas muchos elementos teóricos propios de enfermería como el holismo del ser humano, la satisfacción de necesidades y resolución de problemas, la capacidad terapéutica, el acompañamiento en los procesos de enfermedad y los conocimientos compartidos provenientes de otras áreas que se usan para fines del ejercicio profesional. Las recomendaciones surgen de las propias sugerencias de las ENF entrevistadas, que en su totalidad consideran la educación continuada en las instituciones como la estrategia más importante para el conocimiento de modelos y teorías, y su posterior aplicación en la práctica.

Palabras clave: cuidado de enfermería, disciplina profesional, práctica, proceso de enfermería.

Abreviaturas: UCI, unidad de cuidados intensivos; ENF, enfermera(s).

\section{THEORETICAL ELEMENTS OF ICU NURSING}

\section{Abstract}

This is a descriptive qualitative research that seeks to identify the theoretical elements of intensive care nursing at two hospitals in Bogotá. The study was conducted between July 2006 and November 2007. The sample consisted of nurses who worked in these two hospitals. A semi structured audio recorded survey was designed to obtain information. The number of participants was determined using the saturation principle.

Fecha recibido: febrero 19 de 2008 - Fecha aceptado: abril 23 de 2008

Asesora metodológica: Merideidy Plazas. Docente División de Investigaciones, Fundación Universitaria de Ciencias de la Salud. Bogotá D.C. Colombia.
Asesor temático: José Miguel Díaz. Docente Facultad de Enfermería, Fundación Universitaria de Ciencias de la Salud. Bogotá, D.C. Colombia

* Enfermeras estudiantes de especialización en cuidado intensivo III semestre, Fundación Universitaria de Ciencias de la Salud. 
The results were the answers on nursing care obtained through the interview. Nursing care was defined by the participants as the direct attention delivered to a holistic being to address his needs, mainly physiological needs. Six of the nurses stated they learned about these models and theories during their university training, four cited an author or their own concepts on a theory and only one demonstrated to have basic skills on one of the theories. Three referred total lack of knowledge about the topic. This research reflects that nurses are not using models and theories through a systematic pattern, the delivery of their care is not based on them and very few are interested in their study and application. Nevertheless, we identified many theoretical elements of nursing through the answers to the interview, such as, human holism, addressing human needs, problem solving, therapeutic capacity, follow-up of the disease process and other knowledge shared with related disciplines used in their professional practice. The recommendations resulting from this research are suggested by the interviewed nurses. They consider continuing education programs in health institutions as the most important strategy to update themselves with current knowledge on models and theories and apply them to practice.

Key words: nursing care, professional discipline, practice, nursing process.

\section{Introducción}

El cuidado, luego de un largo proceso evolutivo de la disciplina, ha sido identificado como el objeto de la práctica profesional, el ideal moral y la razón de ser de la enfermería. ${ }^{1}$ Resulta lógico y necesario pensar que debe reflejarse en forma significativa en el pensar y actuar diario de toda ENF, pues solo así es posible validar y reafirmar el nivel de disciplina profesional.

El grupo investigador tiene particular interés en identificar en las ENF asistenciales que se desempeñan en las UCI, los elementos teóricos propios del cuidado que durante décadas el componente disciplinar se ha esforzado en estructurar, validar y difundir en la comunidad de enfermería. Es de gran importancia para alcanzar el objetivo de este estudio, realizar una aproximación y describir cuales son las bases conceptuales que guían la práctica de las ENF en servicio y determinar si se relacionan con el cuerpo propio de conocimientos construido por la disciplina para tal fin, de esta manera se genera un análisis acerca del grado de concordancia entre la teoría y la práctica.

La existencia de un núcleo de conocimientos científicos separados de la práctica, ha llevado a considerar la enfermería como vocación más que una verdadera profesión, situación que ha hecho poner en duda su existencia como disciplina. ${ }^{2}$ Por este motivo es de enorme importancia investigar el estado de la práctica de enfermería, identificar fortalezas, falencias, causas y soluciones que ayuden a comprender el evidente proble- ma de discordancia que existe entre lo académico y lo asistencial.

Es el objetivo de esta investigación contribuir al mejoramiento continuo de la práctica actual a través de una crítica objetiva y la descripción de problemas reales que se identifican en el mismo lugar que se manifiestan, como son los servicios asistenciales. Las alternativas de solución deben provenir después de la acción integrada de las ENF dedicadas a la academia, la docencia, la asistencia, la administración o la investigación; esta es pues una invitación al análisis y la discusión.

\section{Método}

Es un estudio descriptivo cualitativo basado en narraciones detalladas de experiencias, pensamientos y conocimientos propios, relacionados con elementos teóricos del cuidado de enfermería que describen las entrevistadas. Para esta investigación las instituciones seleccionadas fueron dos de Bogotá, de cuarto nivel de complejidad y fuente suficiente de información, por la cantidad de unidades de cuidado intensivo (cuatro) y ENF disponibles. La muestra estuvo conformada por profesionales con experiencia en UCI superior a seis meses que en forma voluntaria aceptaron participar.

La prueba piloto se realizó a tres ENF con el fin de verificar que las preguntas utilizadas fueran de total comprensión para las entrevistadas y a su vez generaran suficiente información y cumplieran con los objetivos de la investigación. Se procedió a la entrevista individual gra- 
bada en audio en el sitio de trabajo de las participantes y la trascripción inmediata de la información, hasta que se obtuvo saturación con nueve entrevistas realizadas.

La guía de la entrevista se elaboró con ocho preguntas base y trece más de profundización, todas de carácter abierto. El procesamiento de la información se realizó a través de codificación y agrupación en cuatro categorías: elementos del cuidado, práctica, proceso de enfermería y utilidad de los elementos teóricos, categorías que el grupo investigador consideró necesarias para abordar y analizar de manera completa el área problema y que adelante se discutirán.

La calidad de esta investigación estuvo determinada por el rigor metodológico, cumpliendo con los criterios de auditabilidad, credibilidad y triangulación teórica. Para ello se diseñó un marco metodológico detallado, específico y secuencial, que describe con exactitud el proceso de esta investigación y permite su verificación en un momento dado. De igual manera la información recolectada es comparable y la discusión se genera a través del análisis con base en la teoría propia de enfermería a través de la triangulación, método que permitió la depuración de la información y la objetividad y confiabilidad en los resultados obtenidos.

\section{Resultados y discusión}

Las nueve ENF participantes laboraban en la UCI, el tiempo de experiencia promedio era de catorce años y el nivel educativo estaba determinado por actualizaciones, seminarios o congresos, y solo una era especialista en UCI. La guía de la entrevista se elaboró con ocho preguntas y algunas de profundización que fueron requeridas con el fin de ampliar la información cuando esta resulto insuficiente. En general hubo adecuado nivel de compresión y gran calidad y variedad en las repuestas que permitió un amplio análisis.

Desde el inicio de la investigación se determinaron cuatro categorías con las cuales se consideraron abordados todos los tópicos de interés relacionados con el área problema y después sirvieron de referente para la clasificación y el análisis de la información. La categoría elementos del cuidado agrupó todos los conceptos acerca de cuidado de enfermería, filosofías, modelos y teorías, con el fin de identificar elementos teóricos propios y otros que fundamentan la práctica de las ENF entrevistadas. La categoría proceso de enfermería, hace referencia al método que emplean las entrevistadas para dar cuidado y los pasos puntuales que siguen, de esta manera fue posible identificar diferencias y similitudes importantes en el pensar y proceder entre una y otra ENF para alcanzar un mismo fin.

La categoría práctica, agrupa las actividades que las ENF entrevistadas identifican como de cuidado dentro de su quehacer diario y fue determinante a la hora de comparar la definición que dan de cuidado frente a las acciones concretas que realizan y el orden de prioridades que establecen. La última categoría, utilidad de los elementos teóricos, trataba de identificar la importancia que le da cada ENF a los elementos teóricos propios de enfermería y el grado de aplicabilidad que le confieren. Quizás fue la más concluyente y en la que hubo mayor grado de similitud en las respuestas. .

\section{Categoría elementos del cuidado}

El cuidado de enfermería se definió por las ENF entrevistadas como la atención directa que se presta a un ser holístico, cuyo fin es la satisfacción de necesidades siendo prioritarias las de origen fisiológico. Otras definiciones menos frecuentes fueron "protección maternal pero con base científica", "poner la tecnología al servicio de un ser humano" y "brindar bienestar total".

Cuando se indagó sobre una definición de cuidado intensivo, lo expresaron como atención directa más personalizada, compleja, de menor participación del paciente y por ende mayor dependencia. Otras características mencionadas fueron "mayor dedicación y vigilancia", "prevención de complicaciones como fin ultimo" y "monitoreo integral".

Es posible identificar dentro de las definiciones obtenidas algunas intervenciones y actividades de las UCI que aunque no son de cuidado, generan confusión en las entrevistadas porque son ellas quienes las ejecutan. También se rescatan elementos teóricos propios del cuidado de enfermería como el holismo, la interacción ENF-paciente, satisfacción de necesidades, capacidad terapéu- 
tica y búsqueda de bienestar. Aunque son conceptos aislados y bien estructurados, están desprovistos de suficiente argumentación teórica. El grupo investigador identificó los elementos teóricos mencionados por las entrevistadas, en algunas filosofías, modelos y teorías de enfermería y constataron que hacen parte del conocimiento vigente de la disciplina.

A pesar de la validación de modelos y teorías para la práctica de enfermería, es evidente el desuso en la muestra objeto de este estudio. Al revisar los presupuestos teóricos que sustentan el concepto de cuidado y sus dimensiones, encontramos que muchos de los autores (Imogene King, Ida Orlando, Joyce Travelbee, Ernestine Wiedenbach, Josephine Paterson y Loreta Zderad) han encontrado que la dimensión interacción es fundamental en la relación ENF-paciente $^{3}$. Otras como Myra Levine y Dorotea Orem han resaltado el valor terapéutico de la intervención de enfermería. ${ }^{3}$ Jean Watson provee una filosofía humanista que profundiza en el holismo y los valores de la ENF, ${ }^{1}$ de donde sin duda se obtienen bases fundamentales para la práctica clínica.

Seis de las ENF refieren haber conocido los modelos $y$ teorías en su formación universitaria, cuatro mencionaron alguna autora o conceptos propios de una teoría y solo una demostró dominio básico de una de las teorías. Tres enfermeras refirieron desconocimiento total sobre el tema "cuando yo estudié, no existían". Las autoras mencionadas fueron Calixta Roy, Dorotea Orem y Virginia Henderson, siendo relacionados conceptos concretos como adaptación, autocuidado y satisfacción de necesidades respectivamente. Algunas entrevistadas presentaron confusión en cuanto a nombres y conceptos de las teoristas, de la misma manera ninguna conocía el significado de lo que es una disciplina profesional, grado actual de enfermería.

En la subcategoría otros elementos teóricos, se agruparon conceptos y saberes que no hacen parte del cuerpo propio de enfermería, sino del conocimiento compartido y que las ENF identifican como necesarios para dar cuidado y fundamentales en su práctica profesional, como fue para siete de ellas la fisiopatología, indispensable a la hora de plantear el plan de cuidados. Otras áreas del conocimiento mencionadas fueron farmacología, psico- logía, ética, gerencia y dos valoraron su experiencia como fuente confiable de conocimiento.

Es difícil reconocer el valor y la importancia del cuerpo de conocimientos propios de la disciplina profesional de enfermería cuando se desconoce su estructura jerárquica, la riqueza y la variedad de sus obras. Por otra parte resulta razonable la forma en que las entrevistadas fundamentaron el uso de conocimientos compartidos como principios de fisiología, patología y farmacología entre otros, como base estructural de la planeación y ejecución del cuidado de enfermería. Lo cuestionable desde el punto de vista científico es que no hagan uso cotidiano del conocimiento que les ofrece su propia disciplina, situación interesante para proponer posteriores investigaciones que permitan enriquecer la relación entre el cuerpo teórico y la aplicación al cuidado del paciente en la labor cotidiana.

\section{Categoría proceso de enfermería}

El proceso de enfermería según las entrevistadas, es el medio para llegar a la elaboración de un plan de cuidado, cuyo paso más importante es una valoración rápida del paciente que permite identificar las necesidades urgentes de acuerdo con el estado fisiopatológico y priorizar las intervenciones. Dentro de este proceso describen como importante el recibo del paciente; en este primer paso las ENF obtienen una información general que les permite rápidamente identificar y ordenar los procedimientos, la logística y las actividades iniciales. Otros pasos mencionados son "presentarse al paciente", "seguimiento y monitoreo", "registro del estado del paciente“"y "evaluación de la evolución del paciente “.

Tres indican que el cuidado es "espontáneo" y "no requiere planeación sino que surge en el quehacer diario", otras afirman que no realizan ningún tipo de registro de su plan de cuidados y algunas refieren que solo anotan en el kárdex las actividades prioritarias. Ninguna mencionó la elaboración o registro de diagnósticos de enfermería, a pesar de su importancia dentro del proceso de la disciplina. Considerando que el diagnóstico es asumido como el razonamiento o aplicación del pensamiento critico para identificar problemas de salud reales o po- 
tenciales, ${ }^{4}$ resulta preocupante que la falta de registro pueda ser la consecuencia de su omisión, implicando para el paciente el no abordaje ni tratamiento holístico, sin identificar los propios problemas y competencias de enfermería para entrar a asumir los ya establecidos por otras profesiones. Por tanto es válido considerar que la formulación de actividades, su ejecución y evaluación deben recuperar la metodología planteada por la disciplina.

Las ENF entrevistadas atribuyen la falta de registro a las múltiples actividades como las de carácter administrativo general, logístico y de mantenimiento que realizan en las UCI, a la complejidad de los pacientes y la necesidad de asistencia directa que demandan demasiado tiempo "uno como enfermera se sienta a hacer planes de cuidado y esas cosas o atiende al paciente". Sin duda un paso definitivo será el reconocer que el registro es la única evidencia tangible del proceso de enfermería y que no realizarlo genera como grave consecuencia el anonimato de su labor.

El proceso de enfermería es un sistema de planificación en la ejecución de los cuidados compuesto de cinco pasos: valoración, diagnóstico, planificación, ejecución y evaluación. ${ }^{5}$ Es un método sistemático, lógico y secuencial claramente estructurado, descrito y aceptado por la comunidad científica. Es enfático en la necesidad de registro escrito y constituye la estrategia metodología de operacionalizacion del cuidado de enfermería. De esta manera resulta preocupante que dicho proceso se lleve a cabo solo de forma parcial y de manera particular por cada enfermera, pues se resta rigurosidad científica.

\section{Categoría práctica}

Más de la mitad de las ENF coinciden en identificar la administración de medicamentos y el monitoreo continuo del paciente como actividades de mayor importancia. Otras mencionadas fueron educación al paciente, cumplimiento de órdenes medicas y aviso de complicaciones, control de líquidos, proporcionar comodidad, aliviar el dolor e intervenciones acordes con patología.

La práctica de enfermería está determinada por tres funciones básicas que se realizan en dimensiones y ámbitos sociales diferentes, ellas son: cuidar la salud, administrar el cuidado de enfermería y educar para la salud. ${ }^{6}$ Las
ENF clínicas entrevistadas sin duda trabajan en el cumplimiento de dichas funciones, sin embargo el cuestionamiento sigue siendo el mismo, ¿son todas estas actividades propias de cuidado de enfermería? Al parecer se han ido sumando un sin fin de actividades que aunque sin duda son prioritarias en la UCI, es evidente el distanciamiento que se genera del verdadero objetivo de la profesión, el cuidado.

\section{Categoría utilidad de los elementos teóricos}

Seis de las ENF manifestaron que no utilizan modelos o teorías de enfermería y no lo consideran indispensable para su práctica profesional, "llevo quince años ejerciendo mi profesión sin conocerlos siquiera". Algunas manifestaron que no son prácticos y resultan demasiado complejos, pero coinciden en la necesidad de "tener claridad conceptual para poder brindar cuidado".

Las ENF de UCI muestran particular interés en fundamentar sus acciones y validar el conocimiento que poseen, con frecuencia dentro de la entrevista argumentan de manera efectiva sus intervenciones desde la fisiopatología, pero hacen escasa o ninguna alusión al conocimiento propio de enfermería, lo que supone que poco se incluye en su práctica profesional. Se percibe cierto desinterés y falta de credibilidad en modelos y teorías; muchas relacionaron su aplicación con la elaboración escrita de planes de cuidado y la realización de lecturas complejas y "poco aplicables a la práctica" que les demanda una cantidad de tiempo importante.

Una de las ENF, especialista en cuidado intensivo, considera que la teoría de la adaptación es aplicable en forma plena al paciente en UCI de cualquier edad; en contraste cuatro manifestaron que ninguno de los modelos propuestos es aplicable a UCI aunque reconocen como insuficiente la información que poseen de cada uno de ellos. No encontrar aplicable ningún modelo o teoría a la práctica clínica en las UCI resulta preocupante, dado que solo con una fuerte argumentación teórica en cada campo en que la enfermería se desempeñe es posible alcanzar la autonomía y la capacidad de desarrollar nuestras propias competencias soportadas sobre bases sólidas, creadas por la profesión y para sus propios fines. 
Adoptar un modelo de enfermería o adaptar uno de los existentes para aplicarlo en un determinado contexto de la práctica es esencial en este momento histórico, cuando se está implementando la gestión del cuidado y se necesita identificar indicadores que permitan medir la calidad del cuidado de enfermería. Resulta infructuoso el intento de avanzar en el desarrollo del componente disciplinar si este, en simultanea, no es reconocido y validado de manera continua en la práctica. De esta manera se establece una relación interdependiente entre las ENF asistenciales y las académicas, relación que con urgencia debe fortalecerse y empezar a producir resultados que devuelvan la confianza a unas y otras, pero que sobretodo propenda por el verdadero desarrollo de enfermería como disciplina y como profesión.

La totalidad de las entrevistadas proponen la educación continuada en las instituciones como la estrategia más importante para el conocimiento de modelos y teorías y su posterior aplicación en la práctica. Otras alternativas son "institucionalización de un modelo o teoría", demostrar la función practica de alguno de ellos en la UCI, "motivación institucional", "abordaje de modelos y teorías en seminarios y actualizaciones" y en general mayor acercamiento del área académica a la asistencial.

\section{Conclusiones}

La conclusión más importante de esta investigación es el hallazgo de una práctica poco provista de elementos teóricos propios, fundamentada en el conocimiento universal de las ciencias de la salud y otras afines, que aunque indispensable no provee elementos teóricos suficientes para el desarrollo de una práctica, autónoma y reconocida. El componente disciplinar y el asistencial se encuentran limitados en su desarrollo por una profunda desarticulación que supone fenómenos complejos y ameritan mayor investigación para su comprensión y resolución. Dicha condición esperamos inquiete y motive a la comunidad de enfermería para la integración en la búsqueda conjunta de estrategias, alternativas y soluciones.

\section{Recomendaciones}

Las recomendaciones producto de esta investigación surgen de las propias sugerencias de las ENF entrevistadas, quienes en su totalidad consideran la educación continuada en las instituciones como la estrategia más importante para el conocimiento de modelos y teorías, y su posterior aplicación en la práctica. El grupo investigador considera que deben buscarse y poner en marcha con prontitud estrategias que posibiliten un acercamiento verdadero de la academia a la realidad de la enfermería asistencial y viceversa; solo así y de ninguna otra manera es posible llevar la teoría a la práctica.

\section{Referencias}

1. Neil R.M. Jean Watson: filosofía y ciencia del cuidado. En: Marriner TA, Alligood MR. Modelos y teorías en enfermería. 5a. ed. Madrid: Elsevier; 2003. p. 145-64.

2. Durán de Villalobos MM, Enfermería: desarrollo teórico e investigativo. Bogotá: Unibiblos; 1998.

3. Alfaro-Levfevre R. Aplicación del proceso enfermero: fomentar el cuidado en colaboración. $5^{\mathrm{a}}$. ed. Barcelona: Masson; 2003.

4. Daza de Caballero R. Cuidado y práctica de enfermería: línea de investigación. Index Enferm. Publicación seriada en Internet 2005 Nov. citado 2008 Jun. 25; 14 (50); aprox $10 \mathrm{p}$. Disponible en: http://scielo.isciii.es/ scielo.php?script $=$ sciarttext $\&$ pid $=$ S 1132 $12962005000200012-\&$ Ing=pt\&nrm=

5. Castrillon MC. La práctica de enfermería como objeto de estudio. Invest. educ.enferm. $1992 \mathrm{Sept} ; 10(2)$ : $15-28$.

6. Reyes J. Jara P, Merino JM. Adherencia de las enfermeras/os a utilizar un modelo teórico como base de la valoración de enfermería. Cienc. enferm. 2007 Jun; 13 (1): 45-57. saturation principle. 